PAPER

\title{
Intelligibility comparison of Japanese speech with competing noise spatialized in real and virtual acoustic environments
}

\author{
Kazuhiro Kondo*, Takahito Chiba ${ }^{\dagger}$, Yuichiro Kitashima ${ }^{\ddagger}$ and Noriyasu Yano \\ Graduate School of Science and Engineering, Yamagata University, \\ 4-3-16 Jonan, Yonezawa, 992-8510 Japan
}

(Received 26 June 2009, Accepted for publication 12 November 2009)

\begin{abstract}
We investigated the effect of a competing noise source on the intelligibility of target speech in various acoustic environments. To spatialize these sources in virtual acoustic space, we used head-related transfer functions (HRTFs) measured using the KEMAR dummy head, as well as HRTFs measured individually for each subject. We also compared the intelligibility when these sound sources were generated from loudspeakers located at the target and competing source positions in real acoustic space. The speech intelligibility of the target speech was evaluated using the Japanese diagnostic rhyme test (DRT). The target speech was placed directly in front of the listener, and a single competing source was placed on the same horizontal plane at various azimuths and distances. Individual HRTFs showed slightly better intelligibility than those with KEMAR HRTFs, but the difference was small in most cases. Intelligibility in real acoustic space still outperformed both types of HRTFs, especially when the competing sound source was closer to the listener than the target speech and the competing source was located at $180^{\circ}$. However, this difference was small in most of the other competing source locations tested.
\end{abstract}

Keywords: Spatialized speech, Speech intelligibility, HRTF, Virtual acoustic space, Loudspeaker sources

PACS number: 43.72.Kb, 43.66.Pn, 43.71Gv

[doi:10.1250/ast.31.231]

\section{INTRODUCTION}

Novel communication systems that can be characterized by multiple-user participation such as social networking services (SNS) are often being introduced owing to user interest in massive "mingling" systems. Most existing systems are mainly text-based, but there is a growing interest in pseudo real-time communication systems, which integrate video and audio conferencing capabilities. Voiscape [1] is an example of such a system. This system integrates both audio and images (still and moving) in a virtual 3-D environment. Avatars indicating participants and sound-generating objects are placed at arbitrary locations in this virtual space. Each participant's speech and sound objects are localized at corresponding locations. The user is free to move around in this space, and the sound image locations are altered according to changes in relative position. The focus of Voiscape is the sound localization of avatar speech and sound objects. Thus, the main concern is

\footnotetext{
*e-mail: kkondo@yz.yamagata-u.ac.jp

currently with Yokote Municipal Hospital

₹urrently with Hitachi Inf. and Commun. Eng., Ltd.
}

that the perceived positions of sound and video objects match in virtual space. The relative distance between the sound objects is not controlled.

Vocal Village [2] is another experimental system that localizes individual speech in horizontally separated locations. It only uses delays, i.e., the interaural time difference (ITD), and gains, i.e., the interaural level difference (ILD) to horizontally laterize the speech sources of each participant. Since only level and time are altered, the speech is localized "within the head," i.e., speech is perceived by the listener to be coming from inside his/her head. This configuration was chosen for its emphasis on real-time working systems. Although it was found that the identification of each speaker's speech improves, the memory of what had been said in the conversations did not improve significantly with simple laterization [3]. This suggests that simple localization with only ITDs and ILDs is not sufficient to enhance the communication of the spoken content, of which intelligibility may be one of the raw measures.

It is well known that speech intelligibility is affected by the location of surrounding competing sounds. There have been many attempts to measure the effect of competing 
noise on the intelligibility of target speech [4-7]. Among these, Hawley et al. have conducted the most extensive investigation to date [8]. They conducted an evaluation of speech intelligibility of phonetically balanced sentences in English with competing speech. These experiments were conducted using loudspeakers placed at actual source locations (in a sound-field room), as well as simulated virtual sources using the head related transfer function (HRTF) of a KEMAR mannequin [9] (KEMAR HRTF). They found that the relative angle between the target speech and competing speech significantly effects intelligibility.

We are focusing on an acoustic conferencing environment in virtual space, where the intelligibility of the primary speaker's speech, with whom the user is currently having a conversation, is kept high [10]. At the same time, the other speakers' speech as well as sound-generating objects are kept in the background, thereby preserving their "presence." This can be accomplished by using sound image localization. Furthermore, we aim to enhance the intelligibility of the speaker of interest (the primary speaker) by detecting the primary speaker and localizing his/her speech directly in front of and close to the user, while other objects are localized away from and to the sides of the user. In previous work [11], we have shown that the intelligibility of target speech can be kept above $70 \%$ if the competing source is placed at azimuths of more than $45^{\circ}$ from the target speech on the horizontal plane. The sound localization in this case was achieved by applying the KEMAR HRTF to the individual sources.

In this paper, we will compare the results for intelligibility tests obtained using KEMAR HRTFs with the intelligibility achievable using the HRTF measured for each individual (individual HRTF), as well as the intelligibility in real acoustic space, i.e., when speech is generated from actual loudspeakers set at various positions. Since it is generally considered that localization using individual HRTFs is more accurate than that using generic KEMAR HRTFs, intelligibility is also expected to be higher. Also, since the separation of actual sound sources in a real environment should be better than that of simulated localized sources, intelligibility with real sources should give us an "upper limit" for the achievable intelligibility. The following experiments are designed to determine the validity of these expectations.

In the next section, the experimental setup of the speech intelligibility experiments is described. This is followed by the intelligibility test results and discussion. Finally, the conclusion is given.

\section{EXPERIMENTAL SETUP}

\subsection{Overview of the Experiment}

We conducted speech intelligibility tests on read
Japanese words with competing noise. The target speech as well as the competing noise was presented in simulated virtual acoustic environments, as well as in real acoustic space. In virtual environments, the speech and noise were localized by convolving monaural sources with the HRTFs measured for KEMAR mannequins, as well as HRTFs measured for each individual. The localized sources were presented over headphones. In real space, the target speech and noise were generated from loudspeakers placed at actual source positions.

\subsection{The DRT Intelligibility Tests}

The diagnostic rhyme test (DRT) intelligibility test uses word pairs that are different only by one initial phoneme as evaluation stimuli. It is an intelligibility test method in which the subject hears one word and is presented a choice of two rhyming words from which the subject must choose one $[12,13]$. Most Japanese syllables are formed by only a vowel or a combination of a consonant and a vowel. The geminate consonant, the Japanese syllabic nasal, and the contracted sound are excluded from the syllables under consideration. In this research, we did not use words that start with a vowel. Therefore, changing one initial phoneme means changing the consonant. Consonants were categorized into six attributes, and intelligibility was measured by these attributes. We chose a word-pair list consisting of 120 words, or 60 word pairs, i.e., 10 word pairs per attribute [14]. Table 1 shows the phonetic taxonomy used in the DRT, as well as example word pairs for each attribute.

The DRT testing is performed semiautomatically using a Perl/Tk script. During the tests, subjects are presented with the word speech for one of the words in the pair. Competing noise is presented simultaneously with the word speech. The DRT words are short two-mora words with silent intervals before and after. Competing noise is presented only during the word speech including the silent intervals. Both words in the word pair are presented

Table 1 Japanese phonetic taxonomy of the DRT.

\begin{tabular}{lll}
\hline Phonetic taxonomy & Classification & Example \\
\hline Voicing & $\begin{array}{l}\text { Vocalic } \\
\text { and nonvocalic } \\
\text { Nasal } \\
\text { and oral } \\
\text { Continuant } \\
\text { and interrupted }\end{array}$ & zai-sai \\
Strident & man-ban \\
Sustention & hashi-kashi \\
and mellow & jyamu-gamu \\
Gibilation & $\begin{array}{l}\text { and acute } \\
\text { Compact } \\
\text { Graveness }\end{array}$ & waku-raku \\
and diffuse & yaku-waku \\
\hline
\end{tabular}




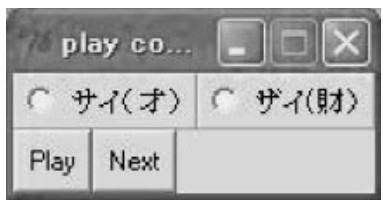

Fig. 1 DRT GUI dialog box. The check button boxes show the two words in the word pair ("sai" and "zai" in this example) from which the listener selects one.

visually on the screen, as shown in Fig. 1, and the subject selects one of the words as the correct word. At this point, the subject can choose to repeatedly hear the same word by repeatedly pressing the "Play" button on the dialog box. When the "Next" button on the dialog box is selected, the next word speech is presented. This procedure is repeated until the predetermined number of words have been presented. The words are presented in random order. The selected word is recorded and processed automatically by a personal computer. The percentage of correct responses is adjusted for chance using the following expression.

$$
\begin{aligned}
& \text { Chance-adjusted percentage correct response [\%] } \\
& \quad=\frac{(\text { Correct responses }- \text { Incorrect responses })}{\text { Total number of responses }} \times 100
\end{aligned}
$$

\subsection{The Stimulus}

The competing noise in all tests was babble noise, i.e., a mixture of speech recorded in a canteen. The noise sample was obtained from the Signal Processing Information Base (SPIB) page [15] of Rice University. This sample was originally recorded at a sampling rate of $19.98 \mathrm{kHz}$ with 16 bits per sample, and was downsampled to $16 \mathrm{kHz}$. The target speech was collected from one female speaker. Ideally, we would have liked to have used more speakers, including male speakers. However, we have already shown that, at least for simple additive noise, little difference is observed between intelligibility for male and female speakers [16]. Moreover, since there was an extremely large number of conditions to test (noise azimuth, distance, presentation mode, etc.), we decided to limit our experiments to one female speaker.

As noted before, we collected 120 words in the DRT test-word set. All sources were recorded at $16 \mathrm{kHz}$ with 16 bits per sample. The noise and speech samples were upsampled to $44.1 \mathrm{kHz}$. The upsampling was necessary to convolve the samples with HRTFs during the spatialization. However, since they were originally sampled at $16 \mathrm{kHz}$, the effective bandwidth was still limited to $8 \mathrm{kHz}$.

\subsection{Source Placement}

This section describes the assumed location of the sound sources within the virtual acoustic space and real space during the DRT tests. All sources were placed on a

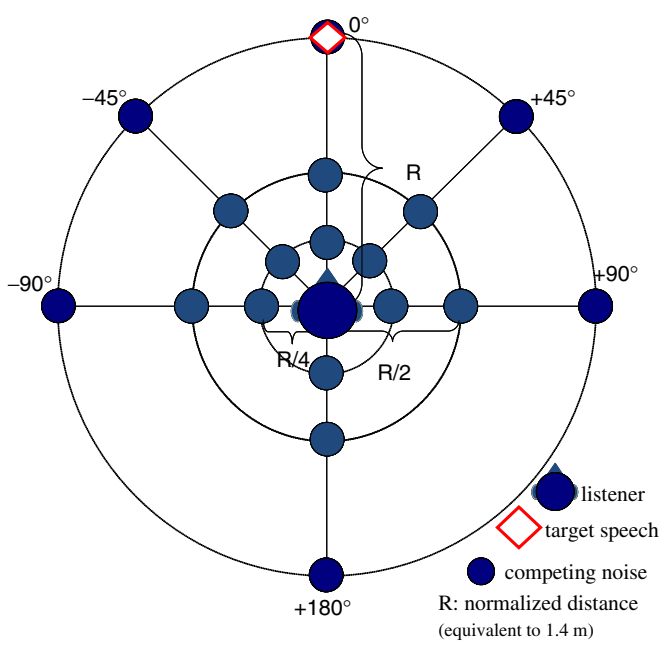

Fig. 2 Placement of target speech and competing noise sources. The distance between the source and the listener was simulated by scaling the level with the reciprocal of the relative distance.

horizontal plane about the height of the listeners' ears when the listeners were in a seated position. The target speech was placed directly in front of the listener at an azimuth of $0^{\circ}$. The competing noise was placed in one of the locations surrounding the listener at azimuths of $0, \pm 45, \pm 90$, or $180^{\circ}$. The noise was placed on one of the three circles centered at the listener's position. The radii of the circles were equal to the target speech-to-listener distance (target speech-to-noise level ratio $0 \mathrm{~dB}$ ), half of the target speechto-listener distance $(-6 \mathrm{~dB})$, and a quarter of the distance $(-12 \mathrm{~dB})$, respectively. The distance in both the virtual space and real space was simulated by adjusting the sound level, i.e., scaling the output sound level with the reciprocal of the relative distance. Since the competing noise is babble, one can still hear individual speech segments. Thus, localized babble was perceived at noise-to-listener distances inversely proportional to the adjusted level, in the same way that the target speech was perceived at distances relative to the adjusted target speech level. Note that we are ignoring the ITD which may vary with distance. However, this condition is the same for real and virtual space since we are simulating distance by only adjusting the sound level in both cases. Thus, this simplification is expected to affect the results in both environments equally. Figure 2 summarizes the source placement.

\subsection{Localization Using HRTFs}

The monaural sources were localized in virtual space by convolution with HRTFs measured for the localized source azimuths. Two types of HRTFs were used: KEMAR HRTFs and individual HRTFs.

The KEMAR HRTFs are HRTF measurements collected and released by MIT using the KEMAR mannequin 
[9]. These measurements were conducted with pinnas of different size on each side. Thus, we used only the right-ear HRTFs in our tests, and used the mirror image of the rightear HRTF for the left ear, as suggested in the readme file provided with the database.

The individual HRTFs were measured at the Research Institute of Electrical Communication (RIEC) at Tohoku University using the HRTF measurement facility in their anechoic chamber. This facility consists of numerous loudspeakers arranged in a circle, both in the horizontal and vertical directions. Time-stretched pulses (TSPs) are played from one of the loudspeakers, which are recorded using tiny microphones embedded at the entrance of both the left and right ear canals of the subject. The circle of loudspeakers is rotated after measurements are made for all loudspeakers on the circle. Measurements are made for all positions surrounding the subjects at all azimuths and elevations. This process is extremely laborious since the subjects must sit still for two hours while the measurements are made. The recordings are converted to HRTFs by applying the inverse TSP filter and then transforming them to the frequency domain.

We measured HRTFs for five students with normal hearing. Intelligibility was tested for all students using their individual HRTFs. Intelligibility was also tested for the five listeners using the KEMAR HRTFs. Some but not all of the listeners were the same with the listeners in the tests using individual HRTFs. Most listeners were "naive" listeners. However, we showed in [17] that training does not affect DRT scores significantly.

Intelligibility for KEMAR and individual HRTFs was measured in separate sessions since there were an enormous number of trials in a session. Within a session, the ordering of the samples by word, noise azimuth, and distance were randomized. The listeners took breaks after approximately $30 \mathrm{~min}$.

The output level of the headphones was adjusted to be roughly equivalent to that of speech played from loudspeakers, as mentioned in the next section.

\subsection{Real Sources Using Loudspeakers}

Intelligibility was also measured for speech and competing noise played from loudspeakers located at actual source locations. Six loudspeakers (all were BOSE MMS-1SP) were placed surrounding the listener in a circle of radius $1.4 \mathrm{~m}$ at azimuths of $0, \pm 45, \pm 90$, and $180^{\circ}$. The height of each loudspeaker was adjusted to match the approximate height of the listeners' ears when the listeners were seated in a chair. The loudspeakers were connected to three amplifiers (all were BOSE 1705II), and the output level was adjusted to approximately $64 \mathrm{~dB}$ for typical speech. The word speech and competing noise were the same as those used in the previous section. The levels of these samples were adjusted and played from the loudspeakers.

All testing was conducted in our acoustic testing room with dimensions of $2800 \times 5423 \times 3610 \mathrm{~mm}$. The walls were lined with sound-insulating sheets and padded with glass-wool panels. The floor was lined with soundabsorption rugs. The doors and windows were covered with sound-insulating curtains. The background noise in this room was approximately $40 \mathrm{dBA}$ during the daytime.

We tested intelligibility in real space with five listeners, all in their early twenties, with normal hearing. Unfortunately, some but not all these listeners the same as the listeners in the tests described in the previous section. The testing for all conditions (target word, noise azimuths, and distances) was performed in a single session but the ordering was randomized. The listeners took breaks after approximately $30 \mathrm{~min}$.

\section{RESULTS}

\subsection{Noise Azimuths vs Intelligibility}

Figures 3-5 show the average chance-adjusted percentage correct response (CACR) for all phonetic attributes with competing noise at the same distance (target speechto-noise level ratio, i.e., SNR $0 \mathrm{~dB}$ ), half the distance (SNR $-6 \mathrm{~dB}$ ), and a quarter of the distance (SNR $-12 \mathrm{~dB}$ ) from the listener relative to the target speech-to-listener distance, respectively, at various azimuths. The target speech was at a fixed location directly in front of the listener. The results shown are the averages for the five listeners. The number of listeners is the same for all following experiments.

Interestingly, these results agree well with prior intelligibility measurements made using similar setups. For instance, Hawley et al. measured intelligibility error rates with one, two, and three competing speeches localized at various azimuths, both in virtual space using KEMAR HRTFs, and in real space [8]. They included data where the target speech was located in front of the listener. They found that, within the $\pm 90^{\circ}$ range they tested, the error rates were minimum at approximately $\pm 90^{\circ}$ and

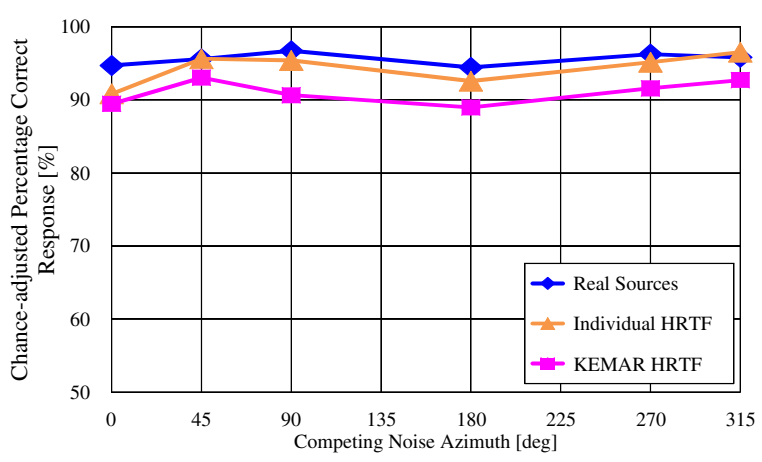

Fig. 3 Competing noise azimuth vs intelligibility $(0 \mathrm{~dB})$. 


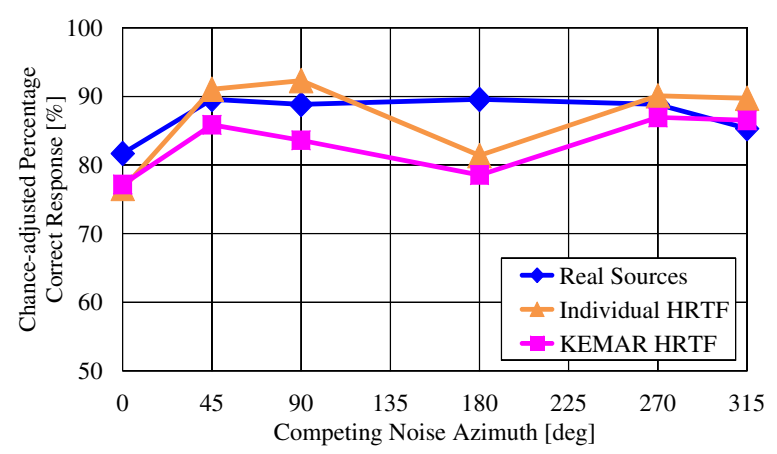

Fig. 4 Competing noise azimuth vs intelligibility $(-6 \mathrm{~dB})$.

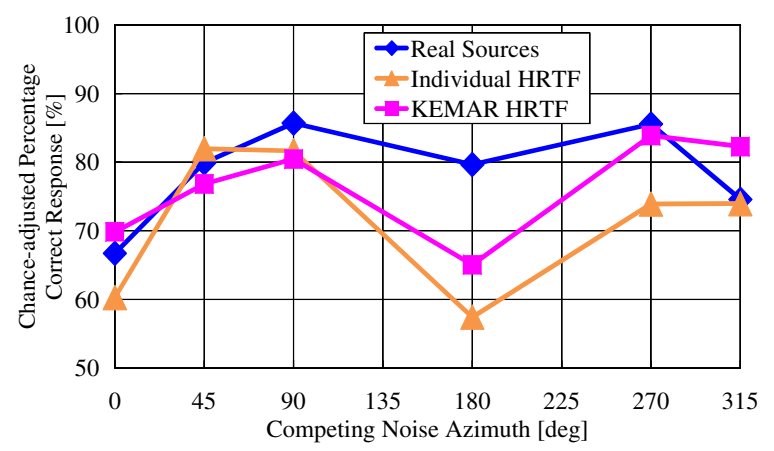

Fig. 5 Competing noise azimuth vs intelligibility $(-12 \mathrm{~dB})$.

maximum at $0^{\circ}$. Since they configured the levels of the target and competing speech to be about the same, the error rates were mostly close to $0 \%$.

Uchida et al. measured the masking level difference of babble noise localized at various azimuths when the target speech was localized in front of the listener [18]. The localization was achieved using individual HRTFs. They found that the masking level difference is largest and almost constant from 45 to $105^{\circ}$, which means that competing noise with a large power is needed to mask the target speech in this range. Their results suggest that higher intelligibility can also be maintained when noise is localized in this range using individual HRTFs.

The comparison between real sources and simulated virtual environments showed higher intelligibility for real sources in almost all cases, as expected. Also, as the SNR decreases, the CACR generally degrades at all noise azimuths. However, the difference in CACR is not large except at noise azimuths of 0 and $180^{\circ}$. The difference is largest at $180^{\circ}$, since for real sources, the CACR does not decrease whereas for virtual sources, the CACR degradation is largest at this angle. This suggests that for a real source, the listeners can separate the target speech in front of them from the noise behind them. For virtual sources, however, listeners confuse the noise behind them with the target speech in front of them, and are not able to separate these two sources.

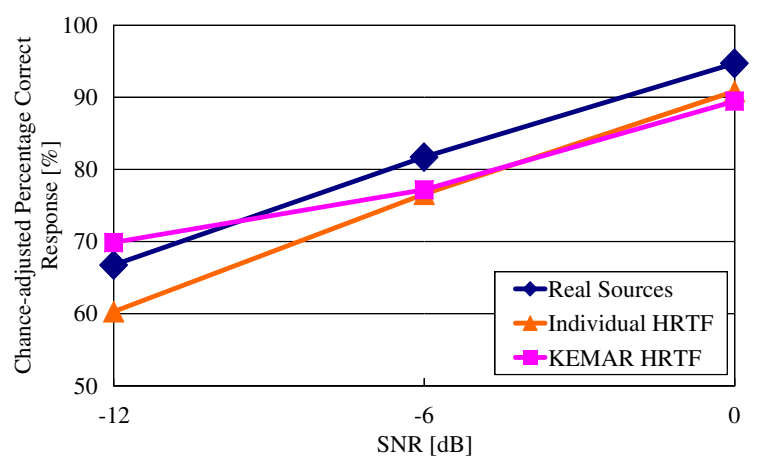

Fig. 6 SNR vs intelligibility (noise azimuth $0^{\circ}$ ).

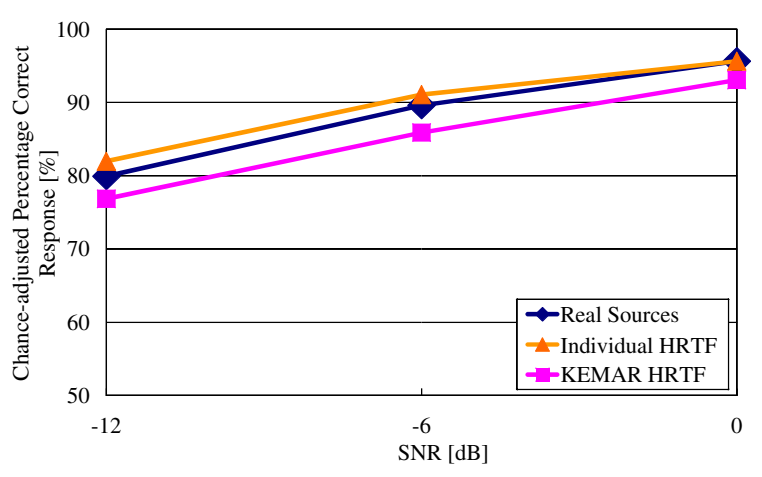

Fig. 7 SNR vs intelligibility (noise azimuth $45^{\circ}$ ).

The two types of HRTFs, the KEMAR HRTFs and individual HRTFs, show no noticeable difference in intelligibility. The individual HRTFs slightly outperform the KEMAR HRTFs when the SNR is relatively high (0 and $-6 \mathrm{~dB}$ ) but degrades to slightly below that of the KEMAR HRTFs at SNR $-12 \mathrm{~dB}$.

\subsection{SNR vs Intelligibility}

Figures 3-5 are replotted in Figs. 6-9 as SNR vs CACR at noise azimuths of $0,45,90$, and $180^{\circ}$, respectively. These plots further highlight the finding that there is no clear difference in the CACR between real and simulated environments except when the noise is at $180^{\circ}$. When the noise is located at this angle, CACR degrades monotonically by about $20 \%$ in simulated environments when the SNR degrades from 0 to $-12 \mathrm{~dB}$, whereas this degradation is only slightly over $10 \%$ for real sources. Also, the CACR degrades by only about $10 \%$ between SNRs of 0 and $-12 \mathrm{~dB}$ when the noise is at 45 and $90^{\circ}$, whereas the degradation is approximately $20 \%$ at 0 and $180^{\circ}$ except for real sources with noise at $180^{\circ}$.

\subsection{Intelligibility by Phonetic Attribute}

Figures 10-15 show the SNR vs CACR for each phonetic attribute when the noise is located at $0^{\circ}$. Only results for real sources and simulated sources using the individual HRTFs are shown since results so far show no 


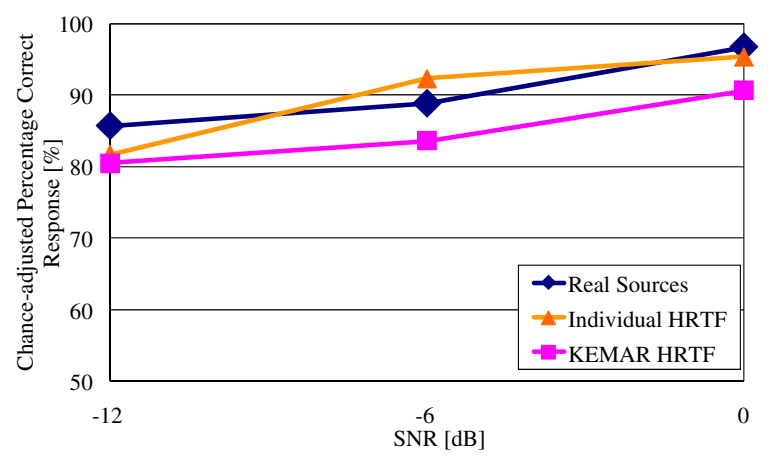

Fig. 8 SNR vs intelligibility (noise azimuth $90^{\circ}$ ).

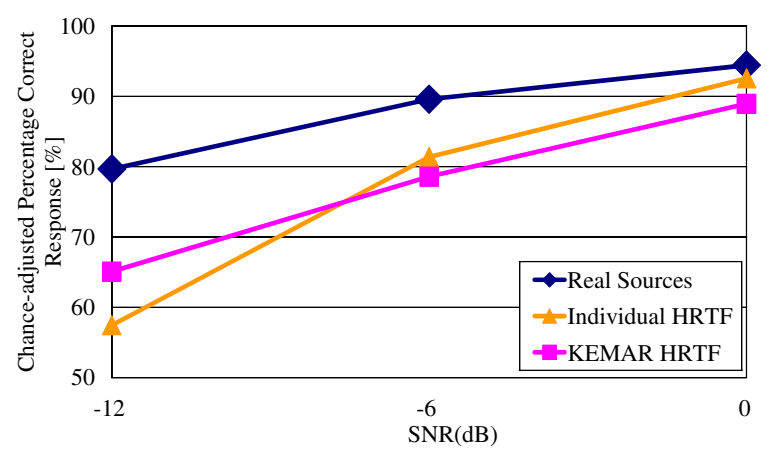

Fig. 9 SNR vs intelligibility (noise azimuth $180^{\circ}$ ).

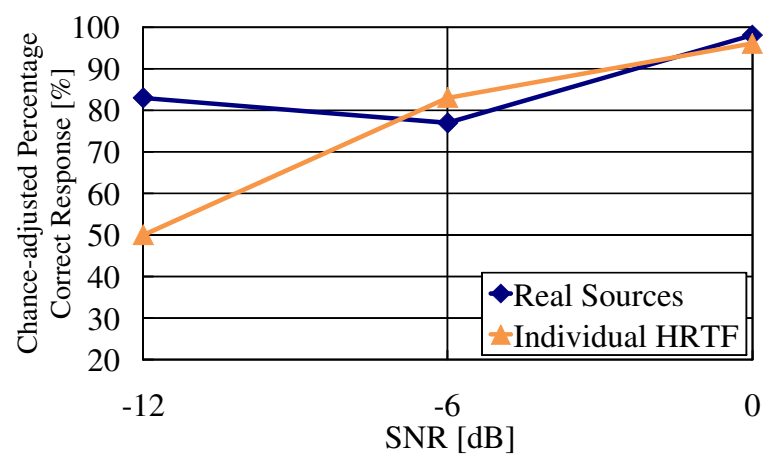

Fig. 10 SNR vs intelligibility (noise azimuth $0^{\circ}$, voicing).

clear difference between KEMAR HRTFs and individual HRTFs, and since we have shown that most phonetic attributes do not exhibit a difference by HRTF type in a limited study [19].

CACR degradation can be classified roughly into three categories. For voicing and sustention, real sources outperform virtual sources when the SNR is poor $(-12 \mathrm{~dB})$ but not when the SNR is above this level. For these two attributes, the CACR for SNR below $-6 \mathrm{~dB}$ does not change for real sources.

For graveness and compactness, the individual HRTFs is lower than that of real sources by a constant amount for most SNRs. For nasality and sibilation, there is no difference in CACR. In fact, sibilation does not seem to be affected by noise at all, remaining constant at about $100 \%$.

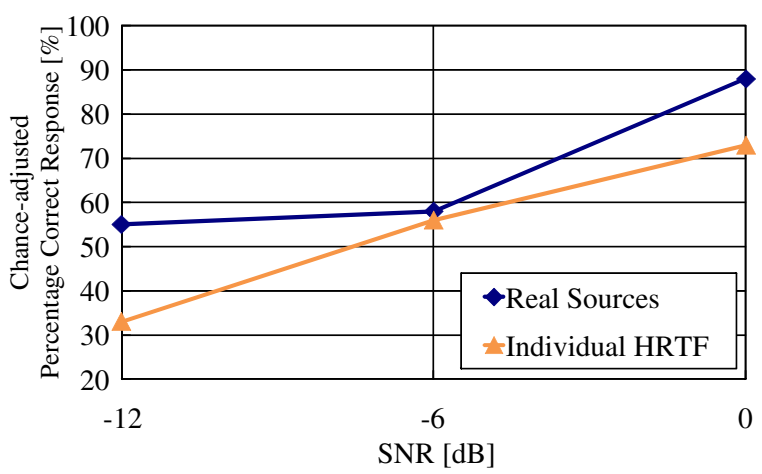

Fig. 11 SNR vs intelligibility (noise azimuth $0^{\circ}$, sustention).

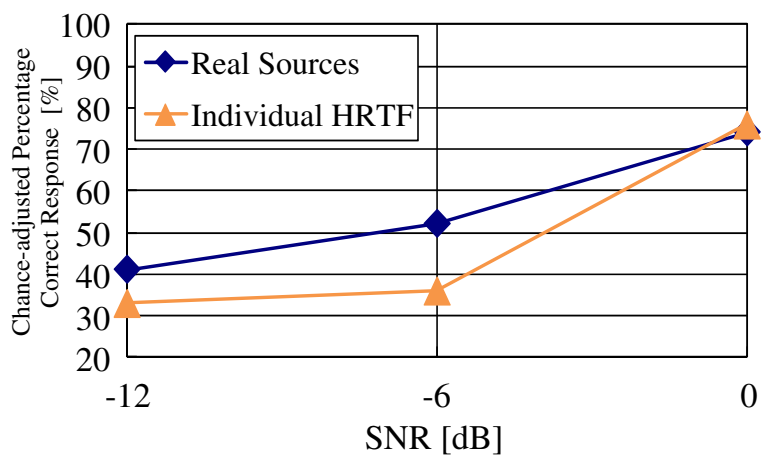

Fig. 12 SNR vs intelligibility (noise azimuth $0^{\circ}$, graveness).

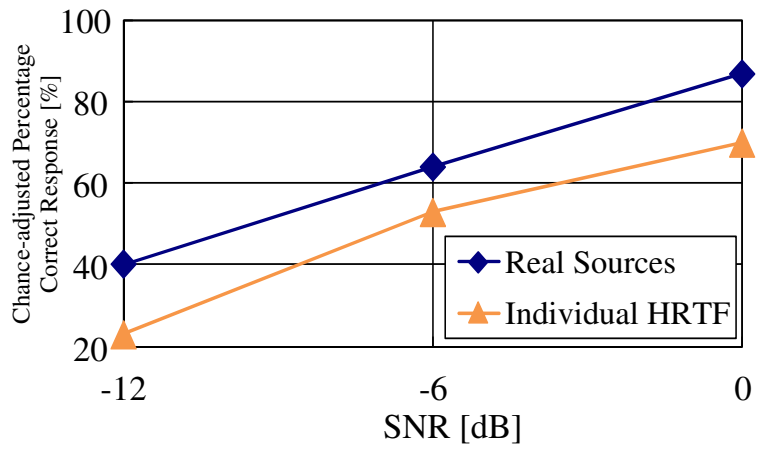

Fig. 13 SNR vs intelligibility (noise azimuth $0^{\circ}$, compactness).

This may be because of the distinct characteristics of the sibilation phones, and also because many sibilation phones, e.g., /s/ and /sh/, have a noticeably higher spectrum than babble noise. This trend has also been reported in [13].

\section{DISCUSSION}

Intelligibility for individual HRTFs and KEMAR HRTFs showed little difference under most of the conditions tested. Thus, it appears that in the multispeaker audio communication systems we are considering, the use of individual HRTFs is not necessary, and generic KEMAR HRTFs will give equally good results. In fact, KEMAR HRTFs will give equally good intelligibility as that obtained when real sources are placed at actual source positions in real space, except when the noise is located 


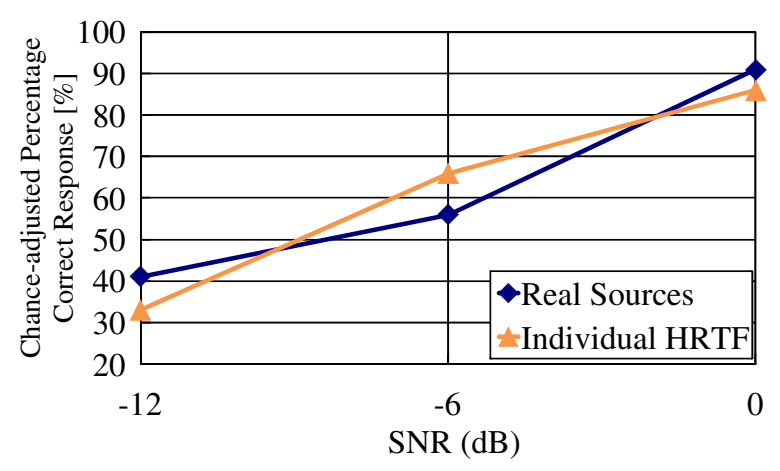

Fig. 14 SNR vs intelligibility (noise azimuth $0^{\circ}$, nasality).

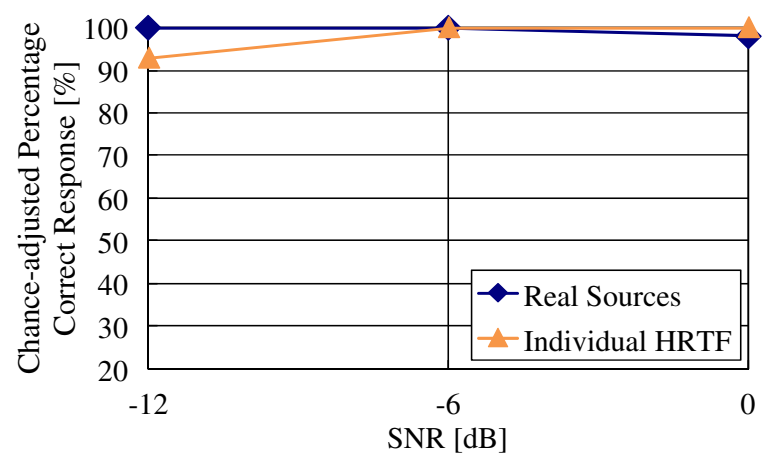

Fig. 15 SNR vs intelligibility (noise azimuth $0^{\circ}$, sibilation).

directly behind the listeners. However, this situation can be easily avoided by limiting the source localization in the front half of the plane. Low intelligibility can also be easily avoided by not localizing the noise sources in the same direction as the target speech and by limiting the level of the noise to be no higher than that of the target speech. All these observations suggest that the design of a multispeaker system with high intelligibility is not particularly difficult. However, these results are valid when all sources are on the same horizontal plane and when the competing source is a single noise source. When there are multiple noise sources, or when the competing sources are speech, the situation may be considerably different. Thus, combinations of other conditions need to be further investigated.

\section{CONCLUSION}

We compared the effect of competing noise sources on the intelligibility of a target speech using real sound sources and in virtual acoustic environments. The intelligibility of the primary target speech when the competing sources were localized at a distance on the horizontal plane was investigated. These sources were spatialized in virtual acoustic space using HRTFs measured using the KEMAR dummy head, as well as HRTFs measured individually for each subject. The target speech was localized directly in front of the listening subject, while the competing babble noise was localized at various azimuths on the horizontal plane. We also compared the intelligibility when these sound sources were actually generated from loudspeakers located at target and competing source positions in real acoustic space. The speech intelligibility of the target speech was evaluated using the DRT. The target speech was placed at a fixed location in front of the listener, while the competing source was placed on the same horizontal plane at azimuths of $0, \pm 45, \pm 90$ and $180^{\circ}$. The distance from the competing source to the listener was set to the same distance as the target speech, half the distance, and a quarter of the distance.

Individual HRTFs showed slightly better intelligibility than those using KEMAR HRTFs when the competing noise was at a greater distance, but KEMAR HRTFs outperformed individual HRTFs when the noise distance was a quarter of the distance of the target speech. Intelligibility in real acoustic space generally outperformed that of both types of HRTFs, especially when the competing sound source was closer to the listener than the target speech. However, this difference was only noticeable at a noise azimuth of $180^{\circ}$ and when the noise was closer to the listener than the target speech.

These results suggest that KEMAR HRTFs are able to achieve intelligibility comparable to that of individual HRTFs or even to sources in real space with a few exceptions. However, these results are only valid for a single competing noise source and when all sources are on the same horizontal plane (no elevation). It is possible that different results will be obtained when the sources have different elevations, when the competing source is speech, or when there are multiple competing sources. Further study is required to investigate these conditions. These additional measurements should indicate the feasibility of spreading the competing sources throughout the 3-D space to retain the high intelligibility of the target speech, which can give more freedom in choosing the placement of various sources according to their relative importance to the listener.

In this study, we have not investigated the effect of head movement on intelligibility. Source location alteration in accordance with head movement (simulating stationary sources) can potentially improve source separation since the listener can use the movement of the relative source location as a clue to help him/her to separate each source. This may improve the intelligibility, and is currently being investigated.

\section{ACKNOWLEDGMENTS}

The authors thank the Suzuki-Iwaya Laboratory of the Research Institute of Electrical Communication at Tohoku University for the use of their facilities and for their support in the measurement of individual HRTFs. This work was supported in part by the Cooperative Project Program of the Research Institute of Electrical Communication at Tohoku University. 


\section{REFERENCES}

[1] Y. Kanada, "Simulated virtual market place by using Voiscape communication medium," Proc. 13th ACM Int. Conf. Multimedia, pp. 794-795 (2005).

[2] R. Kilgore and M. Chignell, "The Vocal Village: enhancing collaboration with spatialized audioconferencing," Proc. World Conf. E-Learning in Corporate, Government, Healthcare and Higher Education (ELEARN) (2004).

[3] R. Kilgore and M. Chignell, "Spatialized audioconferencing: what are the benefits?," Proc. 2003 Conf. Cent. Adv. Stud. Conf. Collab. Res., pp. 111-120 (2003).

[4] A. W. Bronkhorst and R. Plomp, "Effect of multiple speechlike maskers on binaural speech recognition in normal and impaired hearing," J. Acoust. Soc. Am., 92, 3132-3139 (1992).

[5] N. Mesgarani, K. W. Grant, R. Duraiswami and S. Shamma, "Augmented intelligibility in simultaneous multi-talker environments," Proc. Int. Conf. Auditory Display, pp. ICAD03-71ICAD03-74 (2003).

[6] D. Brungart and B. Simpson, "Distance-based speech segregation in near-field virtual audio displays," Proc. Int. Conf. Auditory Display, pp. ICAD01-169-ICAD01-174 (2001).

[7] D. Brungart and B. Simpson, "Optimizing the spatial configuration of a seven-talker speech display," Proc. Int. Conf. Auditory Display, pp. ICAD03-188-ICAD03-191 (2003).

[8] M. L. Hawley, R. Y. Litovsky and H. S. Colburn, "Speech intelligibility and localization in a multi-source environment," J. Acoust. Soc. Am., 105, 3436-3448 (1999).

[9] http://sound.media.mit.edu/KEMAR.html.

[10] Y. Kitashima, K. Kondo and K. Nakagawa, "A speech communication environment using open source software library for active sound image control," Proc. 4th J. Meet. Acoust. Soc. Am. and Acoust. Soc. Jpn., 5pSC46 (2006).
[11] Y. Kitashima, K. Kondo, H. Terada, T. Chiba and K. Nakagawa, "Intelligibility of read Japanese words with competing noise in virtual acoustic space," Acoust. Sci. \& Tech., 29, 74-81 (2008).

[12] W. D. Voiers, "Diagnostic evaluation of speech intelligibility," in Speech Intelligibility and Speaker Recognition, M. Hawley, Ed. (Dowden, Hutchinson \& Ross, Pennsylvania, 1977), pp. $374-387$.

[13] K. Kondo, R. Izumi, M. Fujimori, R. Kaga and K. Nakagawa, "On a two-to-one selection based Japanese speech intelligibility test," J. Acoust. Soc. Jpn. (J), 63, 196-204 (2007) (in Japanese).

[14] M. Fujimori, K. Kondo, K. Takano and K. Nakagawa, "On a revised word-pair list for the Japanese intelligibility test," Proc. Int. Symp. Frontiers in Speech and Hearing Research (2006).

[15] Rice University, "Signal processing information base (SPIB)," http://spib.rice.edu/spib/select_noise.html.

[16] M. Fujimori, K. Kondo and K. Nakagawa, "On low frequencypass characteristics of a one-out-of-two selection-based Japanese intelligibility test," Proc. 6th Tech. Meet. Inf. Process. Soc. Jpn. Tohoku Chapter, A2-2 (2005).

[17] K. Kondo, R. Izumi and K. Nakagawa, "Initial evaluation of a novel Japanese intelligibility test," Tech. Rep. IEICE, SP2000163 , pp. 25-32 (2001).

[18] K. Uchida, T. Nishino, K. Itou, K. Takeda and F. Itakura, "Evaluating the sound localization based on auditory masking," Tech. Rep. IEICE, EA2003-121, pp. 15-20 (2003).

[19] T. Chiba, Y. Kitashima, N. Yano, K. Kondo and K. Nakagawa, "On the influence of localized position of interference noise on the intelligibility of read Japanese words in remote conference systems," Proc. Inter-noise 2008 (2008). 